Politisches Denken · Jahrbuch 1997 
In Verbindung mit der

Deutschen Gesellschaft zur Erforschung des Politischen Denkens

Redaktionsanschriften:

Prof. Dr. Karl Graf Ballestrem

Geschichts- und Gesellschaftswissenschaftliche Fakultät

Katholische Universität Eichstätt

Universitätsallee 1, 85071 Eichstätt

Prof. Dr. Volker Gerhardt, Institut für Philosophie, Humboldt Universität Berlin,

Unter den Linden 6, 10099 Berlin

Prof. Dr. Henning Ottmann

Geschwister-Scholl-Institut für Politische Wissenschaft, Universität München, Ludwigstr. 10, 80539 München

\section{Wissenschaftlicher Beirat:}

Karl Dietrich Bracher (Bonn), Reinhard Brandt (Marburg), Maurice Cranston (London) $(\dagger)$, John Dunn (Cambridge), Iring Fetscher (Frankfurt), Klaus Hartmann (Tübingen) $(\dagger)$, Wilhem Hennis (Freiburg), Dieter Henrich (München), Otfried Höffe (Tübingen), Hasso Hofmann (Berlin), Nikolaus Lobkowicz (Eichstätt), Hermann Lübbe (Zürich), Odo Marquard (Gießen), Kenneth Minogue (London), Michael Oakeshott (London) ( $\dagger$ ), J. G. A. Pocock (Hopkins University), Melvin Richter (New York), Quentin Skinner (Cambridge), Michael Stolleis (Frankfurt) 


\title{
Politisches Denken Jahrbuch 1997
}

\author{
Herausgegeben von \\ Karl Graf Ballestrem, Volker Gerhardt, \\ Henning Ottmann und Martyn P. Thompson
}

Verlag J. B. Metzler Stuttgart • Weimar 
Die Deutsche Bibliothek - CIP-Einheitsaufnahme

Politisches Denken : Jahrbuch...;

Jahrbuch der Deutschen Gesellschaft zur Erforschung des Politischen Denkens.-

Stuttgart / Weimar: Metzler.

Erscheint jährlich.- Aufnahme nach 1991 (1992)

ISSN 0942-2307

1991 (1992) -

Dieses Werk einschließlich aller seiner Teile ist urheberrechtlich geschützt. Jede Verwertung außerhalb der engen Grenzen des Urheberrechtsgesetzes ist ohne Zustimmung des Verlages unzulässig und strafbar. Das gilt insbesondere für Vervielfältigungen, Übersetzungen, Mikroverfilmungen und die Einspeicherung und Verarbeitung in elektronischen Systemen.

ISSN 0942-2307

ISBN 978-3-476-01505-1

ISBN 978-3-476-03678-0 (eBook)

DOI 10.1007/978-3-476-03678-0

(C) 1997 Springer-Verlag GmbH Deutschland

Ursprünglich erschienen bei J. B. Metzlersche Verlagsbuchhandlung und Carl Ernst Poeschel Verlag GmbH in Stuttgart 1997 
Inhalt

REINHARD BRANDT

Europa in der Reflexion der Aufklärung

1

MELVIN RICHTER

Europe and The Other in Eighteenth-Century Thought

25

KENNETH MINOGUE

Popular Sovereignty and the European Community

or: Is »Europe« a Civil Society?

49

PETER NITSCHKE

Europäische Union und die Integration Ost-Mitteleuropas

61

WOLFGANG LEIDHOLD

Tendenzen und Konzepte einer neuen Weltordnung über Prinzipien und Komponenten globaler Ordnungsmodelle 75

DIETER SENGHAAS

Dimensionen einer Weltfriedensordnung

101

Diskussion

STEFAN BREUER

Ein Mann der Rechten?

Thomas Mann zwischen ıkonservativer Revolution`, ästhetischem Fundamentalismus und neuem Nationalismus 
UTE UND HELMUT NICOLAUS

Hofmannsthal, der Staat und die »konservative Revolution«.

Aktuelle Bemerkungen anläßlich einer parlamentarischen Anfrage

141

\section{Rezensionen}

\section{Rainer Forst}

Kontexte der Gerechtigkeit.

Politische Philosophie jenseits von Liberalismus und Kommunitarismus

(Otfried Höffe)

177

Andreas Anter

Max Webers Theorie des modernen Staates.

Herkunft, Struktur und Bedeutung

(Reinhard Mehring)

181

Carl Schmitt

Staat, Großraum, Nomos. Arbeiten aus den Jahren 1916-1969

(Henning Ottmann)

185

Guido Löhrer

Menschliche Würde. Wissenschaftliche Geltung und metaphorische Grenze der praktischen Philosophie Kants

(Norbert Meuter)

187 


\section{Vorwort}

Thematischer Schwerpunkt des Jahrbuchs 1997 ist die internationale Politik. Nicht zum ersten Mal, aber verstärkt in diesem Band, bringt das Jahrbuch die Unruhe zum Ausdruck, die nach der Revolution von 1989 das politische Denken erfaßt hat. Als wie einfach erscheinen rückblickend die Kategorien und Optionen der Politik in einer bipolaren Welt, als wie einfach die Fragen nach der europäischen Identität, solange es um Westeuropa ging. In Wirklichkeit waren die Probleme auch vor 1989 komplex genug, aber es gab Denkschulen, die bewährte Analysen und Antworten parat hatten. Heute spricht man von Globalisierung und Vernetzung, von Weltgesellschaft und neuer Weltordnung, aber auch von Regionalisierung, Ethnisierung und Kampf der Kulturen. Die neuen Begriffe sind Zeichen des Umdenkens, der Suche nach neuen Ebenen der Analyse, nach neuen Ordnungsmodellen. Welche Rolle dabei dem modernen National- und Territorialstaat zukommen soll, ist weiterhin umstritten.

Ein Teil der Beiträge zu diesem Band ist aus Vorträgen hervorgegangen, die im März 1996 auf der Europa-Tagung der Deutschen Gesellschaft zur Erforschung des politischen Denkens in Frankfurt gehalten wurden. Reinhard Brandt und Melvin Richter gehen, aus unterschiedlichen Perspektiven, der Frage einer europäischen Identität im Zeitalter der Aufklärung nach. Kenneth Minogue und Peter Nitschke bereichern die aktuelle Europa-Debatte: der bekannte britische Euroskeptiker Minogue mit Hilfe eines ideengeschichtlichen Kunstgriffs ("what Hobbes, Rousseau and Hegel would have made of contemporary Europe «), Nitschke durch eine kritische Analyse der konzeptionslosen und zögerlichen Politik im Vorfeld der Regierungskonferenz von 1996/97, insbesondere mit Blick auf die Osterweiterung der Europäischen Union. Die Beiträge von Wolfgang Leidhold und Dieter Senghaas gehen über Europa hinaus. Sie sind beispielhaft für den Prozeß des Umdenkens im Bereich der Theorie der internationalen Politik. Beide schlagen neue Kategorien und Dimensionen der politischen Analyse vor, die eine neue Weltordnung denkbar machen.

Auch dieser Band ist nicht ausschließlich einem einzigen Themenbereich gewidmet. Neben den Artikeln zum Schwerpunkt internationale Politik, Europa, neue Weltordnung, wurden zwei Beiträge aufgenommen, die sich auf interessante Weise ergänzen: Stefan Breuer über Thomas Mann sowie Ute und Helmut Nicolaus über Hugo von Hofmannsthal gehen jeweils der Frage nach, in welcher Beziehung ihr Autor zur 'konservativen Revolutionı stand. Wie immer rundet eine Reihe von Rezensionen den Band ab. 